Meta

Journal des traducteurs

Translators' Journal

\title{
Traduire en transparence : génétique d'une version du poème Dunja de Giuseppe Ungaretti
}

\section{Irena Kristeva}

Volume 64, numéro 1, avril 2019

URI : https://id.erudit.org/iderudit/1065332ar

DOI : https://doi.org/10.7202/1065332ar

Aller au sommaire du numéro

\section{Éditeur(s)}

Les Presses de l’Université de Montréal

\section{ISSN}

0026-0452 (imprimé)

1492-1421 (numérique)

Découvrir la revue

Citer cet article

Kristeva, I. (2019). Traduire en transparence : génétique d'une version du poème Dunja de Giuseppe Ungaretti. Meta, 64(1), 145-164.

https://doi.org/10.7202/1065332ar
Résumé de l'article

En 1969, Philippe Jaccottet traduit pour la revue Aquila le poème Dunja de Giuseppe Ungaretti. L'étude tente de relever la singularité de cette traduction, en jetant de la lumière sur la génétique de sa rédaction : autrement dit, de pénétrer le côté invisible du processus traductif auquel collaborent l'auteur et le traducteur. À partir de la clarification terminologique de la notion de transparence, elle s'applique à élucider les stratégies du traducteur, en mettant en relief la dialectique de l'hermétique et de l'herméneutique. La juxtaposition des versions jaccottiennes du poème, élaborées au cours du " work in progress ", renseigne sur les étapes de la traduction transparente, à commencer par le travail sur le lexique qui concerne la recherche d'équivalence. Elle révèle en outre les hésitations et les doutes d'un traducteur averti, conscient de l'impossibilité de comprendre l'énoncé poétique, en négligeant ses aspects affectif, imaginal et prosodique. Pour aboutir à la conclusion qu'il s'agit bien d'une traduction « relevante ", résultat d'un travail minutieux sur les potentialités de la langue cible, qui n’hésite pas à transgresser les normes grammaticales de celle-ci afin de préserver l'étrangeté du texte source. 


\title{
Traduire en transparence: génétique d'une version du poème Dunja de Giuseppe Ungaretti
}

\author{
IRENA KRISTEVA \\ Université de Sofia, Sofia, Bulgarie \\ irena_kristeva@yahoo.com
}

\begin{abstract}
RÉSUMÉ
En 1969, Philippe Jaccottet traduit pour la revue Aquila le poème Dunja de Giuseppe Ungaretti. L'étude tente de relever la singularité de cette traduction, en jetant de la lumière sur la génétique de sa rédaction: autrement dit, de pénétrer le côté invisible du processus traductif auquel collaborent l'auteur et le traducteur. À partir de la clarification terminologique de la notion de transparence, elle s'applique à élucider les stratégies du traducteur, en mettant en relief la dialectique de l'hermétique et de l'herméneutique. La juxtaposition des versions jaccottiennes du poème, élaborées au cours du «work in progress », renseigne sur les étapes de la traduction transparente, à commencer par le travail sur le lexique qui concerne la recherche d'équivalence. Elle révèle en outre les hésitations et les doutes d'un traducteur averti, conscient de l'impossibilité de comprendre l'énoncé poétique, en négligeant ses aspects affectif, imaginal et prosodique. Pour aboutir à la conclusion qu'il s'agit bien d'une traduction «relevante», résultat d'un travail minutieux sur les potentialités de la langue cible, qui n'hésite pas à transgresser les normes grammaticales de celle-ci afin de préserver l'étrangeté du texte source.
\end{abstract}

\section{ABSTRACT}

In 1969 Philippe Jaccottet translated for the magazine Aquila the poem Dunja by Giuseppe Ungaretti. The study aims to highlight the singularity of this translation, shedding light on the genetics of its writing: that is to say to penetrate the invisible side of translating in which both the author and the translator participate. Starting from the terminological clarification of the notion of transparency, it applies itself to elucidate the translator's strategies by highlighting the dialectic of hermetics and hermeneutics. The juxtaposition of the poem's versions, elaborated by Jaccottet during the work in progress, provides information on the stages of transparent translation starting with the lexical work which concerns the search for equivalence. It reveals furthermore the hesitations and doubts of a wise translator, aware of the impossibility of understanding the poetic statement by neglecting its emotional, imaginal and prosodic aspects. In order to reach the conclusion that this is indeed a "relevant" translation, resulting from a meticulous work on the potentialities of the target language, which does not hesitate to transgress its grammatical norms with the purpose of preserving the foreignness of the source text.

\section{RESUMEN}

En 1969, Philippe Jaccottet tradujo para la revista Aquila el poema Dunja de Giuseppe Ungaretti. El estudio tiene como objetivo destacar la singularidad de esta traducción, arrojar luz sobre la genética de su escritura: es decir, penetrar el lado invisible del proceso de traducción en el que participan el autor y el traductor. A partir de la aclaración terminológica de la noción de transparencia, se aplica para dilucidar las estrategias del traductor, destacando la dialéctica de la hermética y de la hermenéutica. La yuxtaposición de las versiones del poema, elaboradas por Jaccottet durante el trabajo en curso, proporciona información sobre los pasos de la traducción transparente, comenzando por el trabajo sobre el léxico que se refiere a la búsqueda de la equivalencia. Revela, además, las indecisiones y las dudas de un traductor advertido, consciente de la imposibilidad de 
comprender el enunciado poético, descuidando sus aspectos emocional, imaginal y prosódico. Para llegar a la conclusión de que se trata de una traducción "relevante", resultado de un trabajo meticuloso sobre las potencialidades de la lengua meta, que no duda en transgredir sus normas gramaticales para preservar la extrañeza del texto fuente.

\section{MOTS CLÉS/KEYWORDS/PALABRAS CLAVE}

cercle hermétique, cercle herméneutique, Jaccottet, traduction transparente, Ungaretti hermetic circle, hermeneutic circle, Jaccottet, transparent translation, Ungaretti círculo hermético, círculo hermenéutico, Jaccottet, traducción transparente, Ungaretti

Traduire serait donc faire œuvre de transparence et de malléabilité au bénéfice d'une œuvre originale qui, elle, serait foncièrement résistance, opacité, voire dureté, lente révélation d'un sens irréductible à une somme de concepts ou d'énoncés.

(Simeone 2014: 31)

\section{Introduction}

«L'extrême brièveté du vers n'a de sens que grâce à une forte accentuation de la langue originale, ce sont de petites sculptures sonores» (Jaccottet 1997: 30). Ces propos de Philippe Jaccottet concernent les premiers poèmes de Giuseppe Ungaretti (18881970), qui inaugurent l'activité créatrice du "poète de la fulgurance $»^{1}$. Le parcours littéraire de l'inspirateur de l'hermétisme poétique italien est jalonné par quelques recueils saillants ${ }^{2}$, à savoir L'allegria (poèmes écrits entre 1914 et 1919), Sentimento del Tempo (1919-1935), Il Dolore (1937-1946), La Terra Promessa (1935-1953) et Il Taccuino del Vecchio (1952-1960). Rédigé en 1969, le triptyque Versi per Dunja réunit une prose brève, Le bocche di Cattaro (Croazia segreta), et deux poèmes, Dunja et $L^{\prime}$ impietrito e il velluto ${ }^{3}$; il représente le chant du cygne de l'homme et de l'écrivain.

Parallèlement, Giuseppe Ungaretti se lance dans des expériences traductives. Il traduit aussi bien des ouvrages d'autrui (Racine, Mallarmé et Saint-John Perse, Shakespeare et Góngora) que certaines de ses propres œuvres. Il lui arrive de pratiquer la traduction à deux voix, en collaborant essentiellement avec Jean Lescure. La traduction française de ses écrits est assumée par des confrères de notoriété considérable, pour ne citer que Francis Ponge, Pierre Jean Jouve et Jean Lescure. Dès les années 1950, Philippe Jaccottet devient son traducteur de prédilection dans la francosphère.

Le poète helvétique fait incontestablement partie de la pléiade d'intellectuels qui ont formé l'horizon traductift dans l'espace culturel francophone au cours de la deuxième moitié du $\mathrm{xx}^{\mathrm{e}}$ siècle. Traducteur chevronné, par métier, par goût et par vocation, il traduit du grec, de l'allemand, de l'italien, de l'espagnol, du russe. Parmi ses innombrables traductions ${ }^{5}$ se détachent Le Banquet de Platon et L'Odyssée d'Homère; Hyperion de Friedrich Hölderlin; Der Tod in Venedig [La Mort à Venise] et Joseph und seine Brüder [Joseph et ses frères] de Thomas Mann; la correspondance entre Rainer Maria Rilke et Lou Andreas-Salomé; l'œuvre intégrale de Robert Musil; Soledades [Solitudes] et les sonnets de Luis de Góngora; et la poésie d'Osip Mandelstam. Cette œuvre-de-traduction (Berman 1995: 121) impressionnante est l'émanation de sa passion pour la poésie:

L'attachement à l'Italie, découverte à vingt ans, juste après la guerre, l'amour de la langue et la proximité des poètes italiens [...] sont évidents: Pétrarque, à la sonorité douce, cristalline, "poreuse»; Le Tasse, étincelant comme Monteverdi; Leopardi, dont il écrit qu'aucun poète n’a su, comme lui, «tirer une limpidité de la tristesse: peut-être 
y fallait-il la langue italienne portée à son point de perfection?». Les contemporains - eux-mêmes, pour la plupart, remarquables traducteurs: de la sombre gravité de Montale à la plénitude sensuelle d'Ungaretti ${ }^{6}$.

Philippe Jaccottet marque immanquablement de sa griffe ses transactions secrètes $^{7}$. En 1950, il traduit pour la revue Rencontre trois poèmes du recueil L'allegria de Giuseppe Ungaretti, rencontré par hasard à Rome en 1946. En 1969, il traduira pour la revue Aquila la petite prose Croazia segreta et le poème Dunja. L'étude se propose de révéler notamment la génétique de sa version de Dunja, en dévoilant le côté non manifeste du processus traductif auquel participent l'auteur et le traducteur. Ses quatre volets s'appliquent à mettre successivement en lumière les pièges terminologiques, les enjeux polysémiques, les stratégies traductives, et la tension entre l'élément hermétique et l'élément herméneutique dans cette traduction transparente.

\section{Tours et détours traductologiques de la notion de transparence}

Je traverse

la distance transparente, et c'est le temps

même qui marche ainsi... (Jaccottet 2009: 269)

La traduction transmet autrement la complexité, les particularités, les nuances de l'original. L'original, en tant que totalité sémantique et formelle, est toujours transcendant par rapport à toute traduction possible. La traduction, en tant qu'une autre compréhension de l'original, est toujours différente par rapport à l'original. Rédigés en langues diverses, l'original et la traduction sont séparés par une distance qu'il est toujours problématique de combler. Pour Philippe Jaccottet, il s'agit bien d'une distance transparente.

Avant de procéder à l'analyse de sa traduction transparente de Dunja, il serait judicieux de clarifier les connotations du concept. Walter Benjamin en parle dans le cadre de la corrélation entre la transparence et la littéralité. Il est convaincu que:

La vraie traduction est transparente, elle ne cache pas l'original, ne l'éclipse pas, mais laisse, d'autant plus pleinement, tomber sur l'original le pur langage, comme renforcé par son propre médium. C’est ce que réussit avant tout la littéralité dans la transposition de la syntaxe; or, c'est elle, précisément, qui montre que le mot, non la phrase, est l'élément originaire du traducteur. Car si la phrase est le mur devant la langue de l'original, la littéralité est l'arcade. (Benjamin 1923/2000: 257; traduction de Gandillac)

La méditation benjaminienne témoigne du besoin inassouvi de l'homme d'un langage supprimant la distance entre les mots et les choses, entre la forme et le sens; d'un langage parfait parce qu'absolu. La traduction transparente n'occulte pas l'original: elle n'est pas sa reproduction mécanique, mais l'harmonisation de son intention. Elle demande la compréhension partagée, l'expérience commune des mondes du texte source et du texte cible, que le traducteur tente d'élargir et d'unifier. Sa visée éthique se réalise le mieux dans l'attachement à la lettre de l'œuvre. La littéralité suppose une sensibilité aiguisée au mot, et par conséquent, exige de traduire non pas proposition par proposition, mais mot pour mot.

Jacques Derrida développe cette idée, en confrontant la littéralité et la liberté. Il prétend qu'il «n'y a que de la lettre, et c'est la vérité du langage pur, la vérité comme langage pur. Cette loi ne serait pas une contrainte extérieure, elle accorde une liberté 
à la littéralité» (Derrida 1987: 235). Cette double orientation par rapport à la lettre et par rapport au sens détermine d'ailleurs les deux modes de traduire qui existent depuis l'Antiquité: verbum pro verbo et sensum de sensu.

Henri Meschonnic déplace, pour sa part, la transparence du texte vers l'état de la langue et le contexte culturel:

Les traductions sont la description du lisible d'une époque et d'une société. Aussi la transparence requise du traducteur n'est autre que le portrait de sa situation dans le langage, en filigrane dans la traduction: l'inscription de ses limites (celles du possible et de l'impossible à dire, selon lui), par son identification à la langue (passive: les autorités) ou au discours, actif. (Meschonnic 1999: 176)

Une fois cernées les limites de la traduction surgit le problème de sa portée. Celle-ci est conditionnée tant par les traductions concrètes et la manière dont elles s'insèrent dans une langue-culture par les formes d'énonciation et de discours, c'està-dire leurs enracinements culturels et linguistiques, que par le processus continu de leur problématisation, c'est-à-dire leurs lectures, relectures et questionnements. Bref, par l'effort de définir et de délimiter leur validité et leur sens.

Rappelons aussi que dans les Belles infidèles, Georges Mounin recourt à la métaphore gogolienne du verre transparent pour distinguer les deux principaux types de traductions :

Ou bien traduire de telle sorte que le texte, littéralement francisé, sans une étrangeté de langue, ait toujours l'air d'avoir été directement pensé puis rédigé en français, c'està-dire, en quelque sorte, réaliser l'ambition des «belles infidèles» sans l'infidélité: première classe de traductions. Ou bien traduire mot à mot de façon que le lecteur, ligne après ligne, ait toujours l'impression dépaysante de lire le texte dans les formes originales (sémantiques, morphologiques, stylistiques) de la langue étrangère, - de façon que le lecteur n'oublie jamais un seul instant qu'il est en train de lire en français tel texte qui a d'abord été pensé puis écrit dans telle ou telle langue étrangère: deuxième classe de traductions. (Mounin 1955/1994: 74)

En s'appuyant sur cette opposition de la traduction transparente et de la traduction colorée, Jean-René Ladmiral forge l'antinomie ciblistes/sourciers: les sourciers «s'attachent au signifiant de la langue du texte-source»; les ciblistes "entendent respecter le signifié (ou, plus exactement, le sens et la "valeur") d'une parole qui doit advenir dans la langue-cible» (Ladmiral 2014: 4). Extrêmement sceptique envers la réduction sourcière du respect du texte original «à une figure éthico-métaphysique de l'altérité» (Rao 2007: 478), il se dresse contre «l'illusion de transparence sourcière [...], l'utopie sourcière de la traduction» (Ladmiral 1998: 980). Les ciblistes sont critiqués à leur tour. Meschonnic rejette ironiquement l'aporie de Ladmiral avec deux arguments: la poétique est à la fois source et cible; la distinction est fondée sur la dichotomie classique du signifiant (la forme) et du signifié (le sens) (Meschonnic 2007: 102-118). En fait, la décision du traducteur n'est jamais ni entièrement sourcière ni entièrement cibliste: elle se situe entre les deux extrémités.

Maurice Blanchot attribue, lui aussi, la qualité de transparence à la traduction qu'il définit diversement:

Il s'agit, bien davantage d'une identité à partir d'une altérité: la même œuvre dans deux langues étrangères et en raison de leur étrangeté et en rendant, par là, visible ce qui fait que cette œuvre sera toujours autre, mouvement dont il faut précisément tirer la lumière qui éclairera, par transparence, la traduction. (Blanchot 1971: 72) 
La dialectique de l'identité et de la différence tend à affirmer la traduction comme une recherche constante de soi-même sous l'impact de l'autre. En quête permanente de l'identité et de la reconnaissance qui implique la participation de l'autre, la traduction contient en puissance le passage d'une langue à une autre. Il y a tout de même lieu de distinguer l'altérité de la différence. L'altérité bloque la compréhension, et donc la traduction. Il est impossible de connaître à fond le «tout autre» (Otto 2001 : 56), la langue étrangère en l'occurrence. On ne la connaît que quand elle est présente dans notre expérience, quand elle entre dans notre langue, c'est-à-dire quand elle perd son étrangeté. La différence, au contraire, cherche la traduction. Elle requiert un travail de déchiffrement, d'intellection, de négociation. L'acte de traduire met en évidence les différences et en même temps les relativise dans les efforts du traducteur de permettre au lecteur de faire face à l'original. La différence produit le sens dans un processus dynamique d'association et de dissociation. Elle relativise la distance entre l'original et la traduction. La «chose» de la traduction n'est pas la «chose» de l'original. Toujours relatif, jamais complètement adéquat, leur rapport ne peut pas être tautologique puisque le texte source et le texte cible sont séparés par une distance.

Par contre, Lawrence Venuti considère la traduction transparente comme «un effet illusoire du discours transparent» (Venuti, 1995: 7, notre traduction) ${ }^{8}$. En raison de la fluidité langagière recherchée, elle se laisse percevoir comme une œuvre rédigée directement dans la langue cible. Le traductologue américain la définit à partir de la distinction entre traduction exotisante (foreignizing translation) et traduction vernaculaire (domesticating translation): à la différence de la «méthode fluide de vernacularisation qui dissimule à la fois le travail du traducteur et les relations asymétriques» (Venuti, 1995: 38, notre traduction) ${ }^{9}$, les «traductions exotisantes, qui ne sont pas transparentes, et qui rejettent la fluidité au profit d'un mélange de discours plus hétérogène, interprètent aussi partiellement le texte étranger, mais tendent à exhiber leur partialité au lieu de l'effacer» (Venuti, 1995: 34, notre traduction) ${ }^{10}$. En supprimant toute trace d'étrangeté de l'œuvre à traduire, le traducteur s'efface en tant que traducteur. Ainsi, Venuti va à l'encontre de l'épreuve de l'étranger, érigée par Berman en principe éthique de la traduction, qui exige de conserver l'esprit de l'œuvre et de la culture étrangère sans craindre de modifier, voire de violer, sa propre langue. L'invisibilité du traducteur résulte, d'une part, de sa décision d'oblitérer sa propre présence. D'autre part, elle pourrait être aussi bien l'indice de la volonté de produire un texte cible qui ne «sent» pas la traduction (pour citer Berman) que celui de l'intention de faire sentir la langue source dans la traduction. L'opposition entre traduction exotisante et traduction vernaculaire n'arrive pas à résoudre cependant ce paradoxe.

Enfin, le traducteur allemand Elmar Tophoven entend par traduction transparente «le passage d'une expression à une autre, la transmutation qui s'accomplit surtout dans la tête, la représentation de ces procédés subtils» (Tophoven 1995: 19). Telle « un work in progress, [elle] donne à lire les étapes de son élaboration: brouillons, premiers jets, bibliographie employée, etc.» (Suchet 2009: 68). C'est bien le cas de la traduction collaborative de Dunja, fruit des efforts conjoints de Jaccottet et d'Ungaretti. Pour cette raison, le concept de traduction transparente sera utilisé dans cette étude, notamment dans l'acception de Tophoven. D’ailleurs, Jaccottet lui-même déclare aspirer à 
[...] une transparence absolue du poème, dans lequel les choses seraient simplement situées, mises en ordre, avec les tensions que créent les distances, les accents particuliers que donne l'éclairage, la sérénité aussi que suscite une diction régulière, un discours dépouillé de tout souci de convaincre l'auditeur, de faire briller celui qui discourt, ou, à plus forte raison, de lui valoir une victoire de quelque espèce que ce soit. (Jaccottet 1957: 120)

Les majeures préoccupations de Philippe Jaccottet restent la prise de recul et la distance critique par rapport à l'œuvre à traduire. Ce détail n'échappe pas à l'œil perspicace de Jean Starobinski: «Jaccottet préfère marquer une séparation, qui n'est pas un désaveu, ni le signe d'une absence de lien passionnel» (Starobinski 1988: 40).

\section{Les arcanes du nom propre}

Pour moi, qui décidément ne comprends pas grand-chose au monde, j'en viens à me demander si la chose «la plus belle», ressentie instinctivement comme telle, n'est pas la chose la plus proche du secret de ce monde, la traduction la plus fidèle du message qu'on croirait parfois lancé dans l'air jusqu'à nous; ou si l'on veut, l'ouverture la plus juste sur ce qui ne peut être saisi autrement, sur cette sorte d'espace où l'on ne peut entrer mais qu'elle dévoile un instant. (Jaccottet 2009: 392-393)

Il incombe à la traduction d'établir des équivalences sur le plan de l'univers et de l'esprit de la langue source et de la langue cible. Or, les mots baignent dans un contexte psychique, culturel et linguistique avec lequel ils interagissent. Transposés dans d'autres contextes, ils perdent l'aura, pour reprendre le terme de Benjamin, de leur univers naturel, et prêtent à confusion. La compréhension du texte à traduire ne suppose pas l'établissement d'une liste d'équivalences, dans la mesure où les univers linguistiques ne coïncident pas et chaque langue structure la réalité à sa propre manière (Humboldt 2006: 81-86). La chose la plus difficile à traduire serait alors l'économie poétique (Derrida 2004: 563), ancrée dans l'univers spécifique d'une langue. Partant du présupposé humboldtien que les mots d'une langue n'ont pas forcément des équivalents dans une autre langue, Derrida estime que la traduction n'est possible qu'à force de renoncer à l'équivalence économique. L'économie poétique risque d'être perdue dans une traduction littérale et d'être déformée dans une traduction trop libre.

Le mot, tant pour Benjamin que pour Derrida, n'est autre chose que le nom, et le nom le point de tangence du langage humain et du langage divin. Limite de la langue, le nom propre s'impose comme limite de la traduisibilité: l'incommunicable dans lequel la parole ne communique rien et le mutisme renferme le Verbe. La tâche du traducteur consisterait justement à donner un nom au muet et à faire taire la voix dans l'indicible pour libérer, dans la traduction, le pur langage enfermé dans l'original. Traduire ou ne pas traduire le nom propre, tel est le dilemme qui se pose à tout traducteur. Et Derrida affirme, d'une part, après Benjamin, que le sacré est intraduisible parce que le sens et la lettre y sont indissociables; et il déclare, de l'autre, que tout est traduisible: «Rien n'est intraduisible pour peu qu'on se donne le temps de la dépense ou l'expansion d'un discours compétent qui se mesure à la puissance de l'original. [...] Mais "intraduisible" demeure [...] l'économie poétique de l'idiome» (Derrida 1996: 100-103). 
L'exemple le plus éloquent d'intraduisibilité, le nom propre, s'avère traduisible grâce à la déconstruction. En proposant un autre langage, celui de la métaphysique, la déconstruction constitue une ressource potentielle pour la traduction. Derrida déconstruit, en effet, $\mathrm{Babel}$ en $\mathrm{Ba}$ (qui veut dire "père» dans certaines langues sémitiques anciennes) et $\mathrm{Bel}$ («Dieu») pour souligner que Babel signifie à la fois la confusion et la cité de Dieu (Derrida 1987: 204). Somme toute, la traduction est, dans un sens transformatif ou productif, une écriture chargée d'effectuer la transposition poétique. Visant à atteindre le pur langage, elle est censée exhumer la chose originaire, voire pré-originaire, contenue dans l'original: «Remonter vers ce qui est pré-originaire [...]. En ce sens, ce serait une traduction, la traduction fondamentale, à partir de laquelle du sens en général pourrait se produire» (Derrida 1982: 178).

La traduction «relevante» (Derrida 2004: 563) devrait repérer les survivances de l'originaire dans le texte source afin d'inscrire dans la langue cible son équivalent le plus pertinent, en utilisant le langage le plus approprié à cet objectif. Pour l'accomplir, Jaccottet a dû réprimer son premier élan de transcrire le prénom croate $\underline{\text { Dunja }}$ comme Dounia, ce qui aurait été justifiable par le désir d'éviter au lecteur francophone non averti la prononciation erronée [dunza]. Après l'échange épistolaire avec Ungaretti, il renonce à cette idée inacceptable pour l'auteur du poème parce qu'elle efface tant le référent que la polysémie du nom.

Les niveaux de traduction ne sont pas juxtaposés ou hiérarchisés, mais interdépendants. Pour cette raison, ils «ne se conçoivent pas indépendamment, notamment dans la phase d'appréhension de l'énoncé original» (Pergnier 1980 : 406). L'ignorance de leur interaction conduit à la sous-estimation du contexte. En «raison de la polysémie de chaque élément linguistique [...] la traduction ne peut faire fi de l'analyse contextuelle» (Pergnier 1973: 36). Une traduction fondée sur le choix aléatoire de l'une des significations répertoriées du mot ne peut pas faire l'objet d'une réflexion critique. Le parcours herméneutique est censé tenir compte de l'enracinement des connotations dans le contexte. La traduction est compromise quand il s'avère impossible de circonscrire dans la signification contextuelle du texte cible des traits distinctifs pertinents pour une situation (Catford 1978: 94).

Les difficultés linguistiques du traduire résultent, entre autres, de la variabilité et de la stratification du langage, et de la relation entre la signification et la désignation. Les obstacles lexicaux découlent de l'ambiguïté et de ses manifestations les plus communes, à savoir l'exponentialité et la polysémie (Catford 1978: 93-103). Dans les deux cas, le contexte joue un rôle décisif pour le parti pris du traducteur. L'exponentialité empêche la traduction. Dans le vers Capricciosa croata notte lucida de Dunja, capricciosa peut se référer aussi bien à la nuit croate qu'à la femme croate. Jaccottet en propose, dans sa première version, deux variantes: Capricieuse nuit croate scintillante et Capricieuse Croate nuit brillante. Ungaretti enlève l'équivocité, en rectifiant le vers traduit puisque, en l'occurrence, il ne s'agit pas d'une homonymie accidentelle, mais d'une ambiguïté référentielle. À ce point, pour éviter la confusion éventuelle, malgré la majuscule de Croate, Jaccottet insère une virgule délimitant les deux unités: Capricieuse Croate, nuit limpide. Un choix quelque peu discutable malgré le consentement de l'auteur, car il altère le jeu initial entre le texte source et son lecteur, en orientant l'interprétation.

Partant de la prémisse que les textes sont des constructions intellectuelles (Bollack 2000: 37), le rapport immédiat à l'œuvre à traduire devient essentiel pour 
sa compréhension. Contrairement au souci de clarification de certaines approches traductives, l'herméneutique critique ne cherche pas la compréhension univoque à tout prix. Dotée d'une précision et d'une diligence particulières, elle ne pousse pas la traduction vers l'illusion de pouvoir comprendre l'incompréhensible; ne vise pas à expliciter l'hermétique et l'opaque dans l'original. Par conséquent, le texte cible devrait conserver les obscurités, les omissions et les obstacles du texte source. Bref, toute sa richesse nuancée. Dans cette perspective, la polysémie précède l'auteur et son œuvre. Bien entendu, l'écrivain puise dans la pluralité sémantique de sa langue, mais il prend sa décision en fonction du sens qu'il veut donner à sa parole, et cette décision devient son intention: "L'auteur tranche, il interprète les associations, mais il y a dans l'écriture quelque chose qui toujours échappe, non qu'il y ait une nécessité ontologique du non-dit, mais parce que le réseau que l'écriture explore est inépuisable» (Bollack 2000: 106).

La polysémie ne repose pas sur l'interchangeabilité universelle des signifiés, autrement dit sur des universaux, puisqu'elle affecte de différentes unités linguistiques dans les différentes langues. C'est un ensemble de valeurs contextuelles instantanées, susceptibles de s'enrichir constamment (Pergnier 1980: 182-189) ${ }^{11}$. Elle n'est pas une caractéristique permanente de la langue, mais une manifestation concrète de la parole dans une situation particulière, une expression de la liberté de l'auteur de coder consciemment ou inconsciemment les éléments du texte source. Le geste du traducteur, autorisé à décider comment recoder ces éléments dans le texte cible, va en amont: il est présumé faire le tri des significations possibles conformément au contexte de production du texte et aux implications sémantiques voulues par son auteur.

L'énoncé étant équivoque, le même signe peut exprimer des concepts différents en fonction du contexte. Le nom propre Dunja en est la preuve. Étant donné que Giuseppe Ungaretti propose un titre polysémique, il serait difficile de déceler toutes les significations de Dunja, en ignorant les artefacts et les référents. C'est un prénom féminin, qui signifie "coing» en croate et "univers» en arabe. Le vocabulaire de l'Islam ${ }^{12}$ oppose Dunya [dunyā], qui désigne l'ici-bas, à l'au-delà, ou Akhira ['âkhira]. Buenodic ${ }^{13}$ précise qu'une forme du mot [dunyā] est «utilisé[e] en hindi, indonésien, malais, swahili, turc et dans presque toutes les autres langues qui font référence au sanscrit ou au Coran, y compris l'arabe», et qu'elle comprend les acceptions de monde ou de terre. Rappelons que Guiseppe Ungaretti est né à Alexandrie et que sa nourrice était croate. S’agit-il de réminiscences de l'enfance? Certainement. À la fin de sa vie terrestre, le poète retourne son regard vers l'Orient de ses origines. Ainsi, la boucle est bouclée.

\section{Les instances de la traduction transparente}

«Mon travail est très intuitif» (Jaccottet 1982a: 10), déclare Philippe Jaccottet à propos de sa traduction de Der Mann ohne Eigenschaften [L'Homme sans qualités] de Robert Musil. On remarque un changement d'attitude envers celle de Dunja: «De l'auteur qu'il admire, et dont il offre le texte, en le tournant vers nous, en version française, il veut que la voix reste perçue, en vérité, distincte comme celle d'un autre» (Starobinski 1988: 40). Résolu d'éviter tout contre-sens et tout contre-ton, mû par la volonté de perfectionner sa traduction, il en rédige trois versions en l'espace de quelques semaines. Ungaretti participe activement au «work in progress»: il lit 
scrupuleusement les variantes, fait des corrections, suggère des solutions. Jaccottet le tient au courant de ses hésitations, le crible de questions, discute ses propositions. Le poème est achevé le 16 juillet 1969. La première version de Jaccottet est datée du 22 août 1969:

TABLEAU 1

Le poème Dunja d'Ungaretti et la première version de la traduction de Jaccottet ${ }^{14}$

\begin{tabular}{|c|c|}
\hline Ungaretti & Jaccottet ( $1^{\mathrm{re}}$ version) \\
\hline Dunja & Dounia \\
\hline Si volge verso l'est l'ultimo amore, & Le dernier amour se tourne vers l'est \\
\hline Mi abbuia da là il sangue & Il m’assombrit de là le sang \\
\hline Con tenebra degli occhi della cerva & Avec les yeux ténébreux de la biche \\
\hline Che se alla propria bocca lei li volga & Qui, les baisse-t-elle sur sa bouche, \\
\hline Fanno più martoriante & Soudain velours, \\
\hline Vellutandola, l'ardere mio chiuso. & Attisent mon feu enfermé ${ }^{15}$. \\
\hline Arrotondìo d'occhi della cerva & Ces yeux arrondis de la biche \\
\hline Stupita che gli umori suoi volubili & Surprise que ses caprices \\
\hline Di avvincere con passi le comandino & Lui enjoignent d'enchaîner \\
\hline Irrefrenabili di slancio. & Avec des pas impétueux. \\
\hline D’un balzo, gonfi d'ira & D’un bond, gonflés de colère \\
\hline Gli strappi, va snodandosi & Ses élans, elle se dégage \\
\hline Dal garbo della schiena & Du galbe de l'échine, \\
\hline La cerva che diviene & La biche qui se change \\
\hline Una leoparda ombrosa. & En panthère ombrageuse. \\
\hline O, nuovissimo sogno, non saresti & Ou ne serais-tu pas le dernier rêve, \\
\hline Per immutabile innocenza innata & Agnelle d'étrange aventure \\
\hline$\underline{\text { Pecorella d'insolita avventura? }}$ & Â l'immuable innocence native? \\
\hline L'ultimo amore più degli altri strazia, & Le dernier amour plus que les autres tourmente \\
\hline Certo lo va nutrendo & Sans doute le souvenir \\
\hline Crudele il ricordare. & L'alimente, cruel. \\
\hline Sei qui. Non mi rechi l'oblio te & Tu es ici. Ne me rendras-tu pas l'oubli \\
\hline Che come la puledra ora vacilli, & Toi qui vacilles maintenant comme pouliche \\
\hline Trepida Gambe Lunghe? & Tremblante Longues Jambes? \\
\hline D'oltre l'oblio rechi & D'outre l'oubli tu me rends, \\
\hline D’oltre il ricordo i lampi. & D’outre mémoire, les éclairs. \\
\hline Capricciosa croata notte lucida & Capricieuse nuit croate scintillante $^{16}$ \\
\hline Di me vai facendo & De moi tu vas faisant \\
\hline Uno schiavo ed un re. & Un esclave et un roi. \\
\hline Un re? Più non saresti l'indomabile? & Un roi? Ne serais-tu plus l'indomptable? \\
\hline
\end{tabular}

Une jeune Croate incite le vieux poète à retourner le regard vers l'Orient. L'incipit renvoie à son enfance passée à Alexandrie en compagnie d'une nourrice croate, et par là, à la Sphinge de Guise, archétype de la mère, de la sagesse et du don oraculaire (Guastella 2003 : 102-108). Son dernier amour est la même femme, à laquelle est dédié tout le triptyque Versi per Dunja. Ungaretti lève lui-même le mystère qui entoure cette figure composite ${ }^{17}$ à connotation universelle dans la prose brève Croazia segreta: 
Quand je perdis mon père en 1890 - je n'avais que deux ans -, ma mère accueillit dans notre maison, comme une sœur aînée, une vieille femme: ce fut ma plus tendre, ma plus experte fée. [...]

J'ai retrouvé Dunja l'autre jour, mais sans les rides dont tout un siècle d'années avait altéré, en les voilant, ses yeux rétrécis; intacts à nouveau ses grands yeux nocturnes, écrins d’abîmes de lumière.

Maintenant, je ne vois plus jamais que Dunja jeune, la très belle, apparaître dans l'oasis, et le désert autour de moi ne me désolera jamais plus, où j'errais depuis tant d'années.

Je n'en doute plus: si Dunja d'abord égare dans les mirages, l'enfant crédule, soudain, devient un enfant croyant, prêt aux libérations que suscitera toujours la vérité de Dunja. «Dunja, chez nous», m'explique le nomade, «signifie l'univers».

Dunja, tu restitues à l'univers ses yeux. (Ungaretti 1969/1973a: 340; traduction de Jaccottet)

L'existence réelle de Dunja perd son importance du moment où elle fait partie de la poétique ungarettienne. L'imaginaire lyrique la dote d'une extraordinaire aptitude à la métamorphose. Tour à tour cerva (biche), leoparda (léoparde), pecorella (agnelle), pouledra (pouliche), elle incarne la transfiguration en puissance: une beauté douce et agressive, docile et farouche, lascive et fulminante. Des quatre images zoomorphiques, c'est leoparda qui paraît la plus problématique à traduire. Vue la proximité du français et de l'italien, Jaccottet prend conscience du risque encouru de percevoir les néologismes et les calques comme une manifestation de maniérisme, «dans lequel compte plus le raffinement de l'opération (lisible par quelques personnes) que l'identité du résultat (doté ou non d'une cohérence et d'une dynamique propres...)» (Simeone 2014: 71). Or, leoparda est un mot rare pour la langue italienne. Et la relecture de sa première version par Ungaretti dissipe ses appréhensions, en rejetant comme inacceptable la substitution de ce signifiant clé du poème par panthère, un équivalent qui aurait détruit le lien entre le mot et son référent. De cette façon, le signifiant est préservé, la valeur sémantique reste suffisamment transparente dans la langue cible, l'effet esthétique est sauvé.

Quand il se heurte à ce genre de difficultés, le traducteur avisé est conscient qu'il ne s'agit ni d'absurdités ni d'erreurs de l'original. Pour transposer cette nouveauté dans sa traduction, il devrait problématiser la norme grammaticale des deux langues. Cette critique intérieure au texte n'est qu'un point de vue provisoire, une contreversion temporaire et limitée. Le grand problème du traducteur ne réside donc pas dans le degré de sa tolérance pour l'intention de l'auteur, mais dans sa capacité de tester la tolérance de la langue cible et sa disposition à accepter des formes insolites qui transgressent sa norme. D’autant plus que le dépassement des usages linguistiques habituels et la reformulation de la propre langue à travers la traduction ne sont nullement faciles et prévisibles, mais dépendent dans une grande mesure de l'aptitude de la langue cible à se soumettre à la force transformatrice de la langue source.

Voilà la version définitive de Jaccottet, confrontée aux corrections (mises en italique) proposées par Ungaretti: 
TABLEAU 2

Traduction du poème Dunja: dialogue Ungaretti-Jaccottet

\begin{tabular}{|c|c|}
\hline $1^{\mathrm{re}}$ version française, corrigée par Ungaretti & Version définitive de Jaccottet \\
\hline Dunja & Dunja \\
\hline Le dernier amour se tourne vers l'est & Vers l'est se tourne le dernier amour \\
\hline Il me noircit le sang & Il m'assombrit de là le sang \\
\hline Avec les ténèbres des yeux de la biche & Par la ténèbre des yeux de la biche \\
\hline $\begin{array}{l}\text { Qui, les baisse-t-elle sur sa bouche, } \\
\text { Rendent plus torturant. }\end{array}$ & $\begin{array}{l}\text { Qui, les baisse-t-elle sur sa bouche, } \\
\text { Rend plus suppliciant, }\end{array}$ \\
\hline De la velouter, mon feu clos $^{18}$ & De la velouter, mon feu clos. \\
\hline Yeux qui s'arrondissent de la biche & Les yeux s'arrondissent de la biche \\
\hline Étonnée que son humeur changeante & Étonnée que ses volubiles humeurs \\
\hline$\overline{\text { Lui enjoignent d'enchaîner }}$ & $\overline{\text { L'obligent à enchaîner }}$ \\
\hline Avec des pas impétueux. & Par l'élan de pas sans frein. \\
\hline D’un bond, gonflé de colère & D'un bond, tout déchirement \\
\hline À chaque déchirure/déchirement, elle se dégage & Rageur, elle se dégage \\
\hline De la souplesse de l'échine, & De la souplesse de l'échine, \\
\hline $\begin{array}{l}\text { La biche qui se change } \\
\text { En léoparde ombrageuse. }\end{array}$ & $\begin{array}{l}\text { La biche qui se change } \\
\text { En léoparde ombrageuse. }\end{array}$ \\
\hline Ou serais-tu rêve inattendu, & Ou, le plus nouveau songe, serais-tu, \\
\hline$\overline{\text { Agnelle d'étrange aventure }}$ & Par l'immuable innocence native \\
\hline À l'immuable innocence des origines? & Agnelle d'étrange aventure? \\
\hline Le dernier amour plus que les autres tourmente & Plus que les autres le dernier amour ravage, \\
\hline Sûrement le souvenir & Sans doute le souvenir \\
\hline Cruellement, le nourrit. & Le nourrit, cruellement. \\
\hline Tu es ici. Ne me rendras-tu pas l'oubli & Tu es ici. Ne me rendras-tu pas l'oubli, \\
\hline Toi qui vacilles maintenant comme pouliche & Toi qui vacilles à présent comme pouliche \\
\hline Anxieuse, Longues Jambes? & $\underline{\text { Craintive, }}$ Longues Jambes? \\
\hline D'outre l'oubli tu me rends, & D'outre l'oubli tu me rends, \\
\hline D’outre mémoire, l'éclair. & D’outre mémoire, les éclairs. \\
\hline Capricieuse Croate nuit $\underline{\text { lucide }}$ & Capricieuse Croate, nuit limpide, \\
\hline De moi tu vas faisant & Tu fais de moi \\
\hline Un esclave et un roi. & 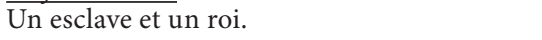 \\
\hline Un roi? Ne serais-tu plus l'indomptable? & Un roi? Ne serais-tu plus l'indomptable? \\
\hline
\end{tabular}

La juxtaposition des versions du poème renseigne sur les étapes de la traduction transparente à commencer par le travail sur le lexique et la prosodie, qui concerne la recherche des correspondances sémantiques et sonores. Elle dévoile les hésitations et les doutes d'un traducteur averti. Philippe Jaccottet n'est certainement pas un Pierre Ménard réécrivant à la lettre l'œuvre originale. Il ne se livre pas à une reproduction mécanique de Dunja. Toutefois, il estime qu'il faut «traduire, dans la mesure du possible et sans tomber dans l'absurde, selon la lettre même du texte» (Jaccottet 1982b: 409). Ainsi, il s'attaque au mot et à la signification dont celui-ci est porteur dans le souci de préserver la distance transparente entre le poème source et le poème cible. 


\section{Cercle hermétique vs cercle herméneutique}

«Le traducteur qui, sans faire un seul contresens, tue le chant, est un malfaiteur» (voir note 6). Pour traduire la poésie hermétique d'Ungaretti, Jaccottet adopte une stratégie herméneutique. Cette herméneutique traductive respecte le style et l'expression spécifiques de l'auteur; observe le rythme et la versification de l'œuvre; reproduit les jeux de langage, en cherchant à circonscrire les « réseaux signifiants sous-jacents » (Berman 1999: 61-62); évite les interactions sémantiques qui n’y figurent pas; bref, tente de restituer l'original avec toutes ses omissions, contrariétés, nouveautés, voire éléments grammaticalement inacceptables. Il ne faut cependant pas oublier que si l'herméneute interprète les textes afin de les comprendre, le traducteur doit comprendre les textes afin de les traduire.

Depuis Schleiermacher, l'herméneutique distingue la traduction (Übersetzen) de l'interprétation (Dolmetschen), en relevant le nouveau rapport au langage, unique en son genre, instauré par la traduction. La traduction présuppose la compréhension du texte à traduire et la perception de la sensibilité linguistique de son auteur, tandis que l'interprétation vise l'explication du texte et l'élucidation de ses ambiguïtés. L'interprétation se déroule sur le plan des apparences et des truchements de l'oral, la traduction implique la connaissance approfondie et l'entendement subtil de la pensée de l'auteur et de la sensibilité de son langage. L'interprétation sert aux enjeux de la communication, la traduction ouvre un horizon culturel par la présentation d'un modèle de connaissance. Du reste, le traducteur ne devrait pas se contenter de communiquer sa compréhension du texte, mais essayer de transmettre l'insaisissable, le mystérieux, le poétique qu'il contient:

Pour que ses lecteurs puissent comprendre, ils doivent saisir l'esprit de la langue qui est la langue natale de l'écrivain, ils doivent pouvoir intuitionner sa façon de penser et de sentir; et pour parvenir aux deux choses, il ne peut leur offrir que sa propre langue, qui ne coïncide jamais pleinement avec l'autre, et que lui-même, avec sa connaissance plus ou moins claire de l'auteur, avec l'admiration et l'approbation plus ou moins grande qu'il lui voue. (Schleiermacher 1813/1999: 45, traduction de Berman)

On traduit avec la conscience que le texte cible, fondé sur l'interprétation, serait toujours imparfait par rapport au texte source, dans l'incertitude d'avoir bien compris la pensée de l'auteur et d'avoir saisi l'esprit de l'autre langue. D’où la nécessité constante de retraduire. La concordance du tout et de la partie s'impose alors comme le critère de la compréhension du texte. Schleiermacher a révélé la double constitution du cercle herméneutique: objective ou grammaticale; subjective ou psychologique. Ces deux aspects de l'interprétation qui caractérisent la circularité de la compréhension sont très importants pour l'acte de traduire. L'interprétation objective se réalise grâce à la méthode comparative qui poursuit une analyse comparée des significations de tel ou tel mot pour déterminer son sens contextuel. L'interprétation subjective essaie de deviner son sens concret à partir de ses usages particuliers par un auteur (Kristeva 2009: 101).

Du point de vue herméneutique, la traduction relève de l'expérience du monde et du rapport à l'autre: elle est une expérience existentielle et dialogique, fondée sur la compréhension de la pensée de l'autre à travers son discours. La traduction est bipolaire: l'identité et la différence y cohabitent, en s'opposant, sans chercher pour autant à s'assimiler réciproquement. Conçu comme un problème essentiel de la tra- 
duction, le cercle dans lequel sont pris le même et l'autre devient le moteur de l'herméneutique, et par là, de la réflexion sur la traduction. Et comme traduire est de l'ordre du sens, la réflexion herméneutique sur l'acte de traduire ne doit pas être négligée. D’autant plus que l'herméneutique et la traduction partagent un objectif commun: la compréhension des textes.

Le sens qui circule dans les textes ancrés dans une tradition culturelle ne provient pas de la langue, mais surgit du langage, de l'usage singulier des mots dans le texte. La traduction affronte des expériences langagières particulières et non pas des structures immanentes à la langue. Mais ces expériences reflètent des visions du monde qui dépassent ce qu'un texte signifie stricto sensu: "Ce qui est finalement à comprendre dans un texte, ce n'est ni l'auteur et son intention présumée, ni même la structure ou les structures immanentes au texte, mais la sorte de monde visé hors texte comme la référence du texte» (Ricœur 1977: 38). Pour Ricœur, le traducteur doit appréhender la dialectique entre l'enfermement et l'ouverture du texte dont le contenu est dirigé vers quelque chose qui va au-delà de son espace: son référent. Par conséquent, la survie de l'œuvre dépend de l'actualisation, par ses lectures, de la chose dont elle parle. Le travail de mémoire fait vivre l'œuvre, en communiquant son sens produit par l'appropriation de la tradition de sa lecture. L'ouverture du texte vers son référent devient la condition de son interprétation:

Transmettre suppose une "place dans le monde», d'où s'exerce une fonction, en tout cas une opération, une mise en acte. L'écriture ne peut supporter, semble-t-il, d'être tout entière dans un réel sur lequel elle aurait prise, ou dont elle participerait en son essence. Toujours elle se berce de l'espoir - de l'illusion motrice? - qu'en elle se concrétise une part impossible, une ligne de fuite, une asymptote. Le seul territoire qu'elle éprouve et revendique n'est-il pas celui de l'aporie? (Simeone 2014: 7)

Benjamin impose les antinomies complémentaires fidélité/liberté et transparence/ littéralité (Benjamin 2000 : 256-260) comme les critères d'évaluation de toute traduction. Si la fidélité s'exerce par respect du langage de l'œuvre à traduire, la liberté s'exerce au nom de l'amour pour le pur langage. Le traducteur devrait donc chercher à atteindre le noyau intraduisible, à saisir l'insaisissable, à capter le souffle invisible de l'œuvre. Il s'ensuit qu'on ne traduit pas purement et simplement pour apporter au lecteur un texte qu'il n'est pas capable de lire dans sa langue originale. En effet, ce n'est qu'en enlevant de la traduction tout ce qui concerne la communication de la signification, qu'on peut accéder à l'intraduisible dans l'original, à la parcelle de pur langage.

Transmettre suppose aussi fidélité et devoir. Deux mots qui contraignent. Si fidélité il y a dans l'écriture, c'est envers une polysémie essentielle, pour ne pas dire une ambiguïté. Si devoir il y a, c'est d'œuvrer à la communauté par la voie paradoxale de la solitude, aux frontières périlleuses de l'hermétisme et de l'extrême délégation... (Simeone 2014: 8)

La fidélité formelle de la version de Jaccottet se manifeste sur le plan de la versification. Quant à la liberté, on note quelques écarts volontaires pour des raisons prosodiques, tels que: la biche qui se change en léoparde au lieu de qui devient; d'outre mémoire, les éclairs au lieu de souvenir, le mot déjà employé, dans la cinquième strophe, comme équivalent de ricordare. Fondé dans le système linguistique de son auteur, le texte dépend à la fois de l'identique et du différent. Dans cette perspective, 
l'acte de traduire est une réponse à l'autre qui ne se réduit pas à la transmission des signes linguistiques, à l'autre qui doit être entendu et à qui doit répondre l'acte de langage du sujet traduisant (Petrilli 2001: 12-16). La difficulté à traduire un texte réside moins dans le fait de devoir traduire ce qui est dit par une langue dans une autre langue que dans celui de comprendre ce qui le rend possible et significatif comme réponse. En appelant à l'intertextualité, le texte véhicule plus de rapports que ceux instaurés par lui-même. Et on n'arrive à comprendre l'énoncé poétique qu'à l'aide d'éléments dont la nature n'est pas purement linguistique, mais aussi affective, imaginale, sonore et rythmique.

Les problèmes communicatifs de la traduction sont engendrés par la pluralité des connotations supposées par les énoncés linguistiques. Mais comme le traducteur est censé rendre aussi bien les émotions et les sensations qu'ils portent, il effectue à la fois un transfert sémantique et un transfert affectif afin d'assurer l'expression apparente du texte et son souffle invisible. Autrement dit, au «transfert des mots et des rythmes, s'ajoute celui des zones irréductibles où l'original est légitimé par sa limite et par ses apories» (Simeone 2014: 40). En l'occurrence, la connotation affective du substantif leoparda, sans doute unique en tant qu'individuelle, doit parvenir impérativement dans le texte cible, en assumant le risque de l'hermétisme.

La musique singulière de Dunja met à l'épreuve son traducteur. Sa transmission révèle un parti pris syntaxique qui jette un défi aux contraintes du français, en optant pour des inversions inhabituelles au sein du vers (Si volge verso l'est l'ultimo amore - Vers l'est se tourne le dernier amour) ou de la strophe (Certo lo va nutrendo / Crudele il ricordare - Sans doute le souvenir / Le nourrit, cruellement).

La stricte observation des enjambements y contribue aussi:

1) Con tenebra degli occhi della cerva Che se alla propria bocca lei li volga Fanno più martoriante Vellutandola, l'ardere mio chiuso.

(Ungaretti 1969c: 325)

a) Par la ténèbre des yeux de la biche Qui, les baisse-t-elle sur sa bouche, Rend plus suppliciant, De la velouter, mon feu clos.

(Ungaretti 1969/1973b : 341-342; traduction définitive de Jaccottet)

2) O, nuovissimo sogno, non saresti Per immutabile innocenza innata Pecorella d'insolita avventura?

(Ungaretti 1969c: 325)

a) Ou, le plus nouveau songe, serais-tu, Par l'immuable innocence native Agnelle d'étrange aventure?

(Ungaretti 1969/1973b: 341-342; traduction définitive de Jaccottet)

Lorenzo Flabbi remarque avec justesse que la dominante phonétique du vers ungarettien est marquée par les «o » qui créent l'effet du cercle hermétique. La deuxième strophe en offre l'exemple le plus éloquent: 
3) Arrotondìo d'occhi della cerva

Stupita che gli umori suoi volubili

Di avvincere con passi le comandino

Irrefrenabili di slancio.

(Ungaretti 1969c: 325)

a) Les yeux s'arrondissent de la biche

Étonnée que ses volubiles humeurs

L'obligent à enchaîner

Par l'élan de pas sans frein.

(Ungaretti 1969/1973b : 341-342; traduction définitive de Jaccottet)

La circularité expressive et la plénitude sonore du substantif italien occhi ne sont pas ressenties dans l'équivalent français. Parfaitement conscient de cette perte, Jaccottet la compense, en la transposant:

[...] en un réseau dense de sifflantes, de sorte que l'idée de la rondeur est donnée non pas par la stase de la circonférence, mais par le mouvement sinueux du cercle en formation. C'est ainsi que le phonosymbolisme est imité, sauvegardé, translaté. (Flabbi $2006^{19}$; notre traduction ${ }^{20}$ )

Son travail sculptural sur le signifiant de l'hapax leoparda ombrosa, le souci d'en mouler la rotondité, d'en augmenter le volume visent à atteindre un effet aussi bien sémantique que sonore. Le leitmotiv des yeux réapparaît dans le tout dernier poème d'Ungaretti, L'impietrito e il velluto, dont les vers Il velluto croato / Dello sguardo di Dunja et Il velluto dello sguardo di Dunja / Fulmineo torna presente pietà, mettant cette fois-ci en valeur la douceur du regard de la belle femme, sont rendus par Jaccottet avec quelques déplacements syntaxiques: Le croate velours / Du regard de Dunja et Le velours de ses yeux revient, / Foudroyante pitié (Ungaretti 1970/2005: 328-329). Ainsi, le «relatif échec de la traduction en tant que transfert et transparence devient condition de son existence autonome, de sa dignité créatrice» (Simeone 2014: 36).

\section{Conclusion}

La signification de l'œuvre littéraire ne se réduit pas à l'intention de son auteur, quelque explicite qu'elle soit. D'où le danger de le consulter outre mesure. Cela ne signifie nullement de privilégier l'intention du traducteur. Cela ne signifie pas non plus que la distance entre l'auteur et le traducteur soit insurmontable. Au contraire, le travail de l'interprétation peut être facilité si le traducteur arrive à repérer l'écart entre ce qui est fixé dans le texte et ce qu'on dit sur le texte.

L'idée de la restitution totale de l'œuvre littéraire dans une autre langue est utopique. La traduction parfaite n'existe pas tout comme le pur langage de Benjamin ou l'absolu littéraire des romantiques allemands. Bref, comme n'importe quel idéal régulateur qui joue pourtant un rôle fondamental dans le développement de la langue ou de la littérature. Il existe, en revanche, une traduction «relevante» qui prend en considération l'étrangeté et la sensibilité de l'original. C'est bien le cas de la version jaccottienne de Dunja, qui prouve que la traduction

[...] ne consiste pas à simplement transmettre dans la transparence, qu'elle n'est pas un pur passage, mais toujours un travail sur sa propre langue, une chance donnée à celleci de remettre en cause ses certitudes et ses limites à travers l'irruption dans son espace d'œuvres et d'écritures étrangères. (Simeone 2014: 21) 
La modestie de Jaccottet qui se prononce sur l'aboutissement de ses efforts de traducteur est exemplaire: «Hors de trop rares miracles, tout traducteur sait que son travail est indéfiniment perfectible» (Jaccottet 1973: 331). Son honnêteté l'est peutêtre davantage: "Devenir des mots, pour les choses, c'est aussi "paraître gagner un sens” (on n'en saurait dire plus sans tricherie)» (Jaccottet 2015: 290). Il confesse à son éditeur «qu'il n'est ni convaincu par cet "étrange poème", ni satisfait de sa traduction». Ungaretti, ne doutant ni de son poème, ni de sa version française, tranche: «La traduction me paraît déjà excellente» (Graf 1998: 64).

\section{NOTES}

1. AmANECER, François (Dernière mise à jour: 6 avril 2014): Ungaretti, ou la fulgurance à l'envers. Recours au poème. Consulté le 17 septembre 2017, <https://www.recoursaupoeme.fr/ungaretti-oula-fulgurance-a-lenvers/>.

2. Voir l'Annexe 1 pour les références bibliographiques des œuvres d'Ungaretti.

3. Ungaretti commence à rédiger le poème L'impietrito e il velluto dans la soirée du 31 décembre 1969 et le termine au matin du $1^{\text {er }}$ janvier 1970. D'abord publié seul chez Grafico Romero, le poème est ensuite ajouté à la deuxième édition (1970) de l'anthologie ungarettienne Vita d'un uomo: tutte le poesie (publiée pour la première fois en 1969).

4. Antoine Berman définit l'horizon traductif comme l'«ensemble des paramètres langagiers, littéraires, culturels et historiques qui "déterminent" le sentir, l'agir et le penser d'un traducteur" (Berman 1995: 79).

5. Voir l'Annexe 2 pour les références bibliographiques des œuvres et des traductions de Jaccottet.

6. Petillon, Monique (21 mars 1997): Les «transactions secrètes» de Philippe Jaccottet. Le Monde des livres. 4.

7. L'expression transaction secrète appartient à Virginia Woolf: «Was not writing poetry a secret transaction, a voice answering a voice?» (Woolf 2008: 306). Philippe Jaccottet la reprend comme titre de ses «lectures de poésie» (Jaccottet 2015).

8. «[...] an illusionistic effect of discourse.»

9. «[...] fluent domesticating method that masks both the translator's work and the asymmetrical relations - cultural, economic, political - between English-language nations and their others worldwide.»

10. «[...] foreignizing translations that are not transparent, that eschew fluency for a more heterogeneous mix of discourses, are equally partial in their interpretation of the foreign text, but they tend to flaunt their partiality instead of concealing it.»

11. Maurice Pergnier développe la dialectique des significations "contextuelles» et "situationnelles", en mettant en rapport les premières avec la polysémie, et les secondes avec la signification. Il émet en outre deux hypothèses sur la polysémie: phénomène chaotique ou manifestation d'un ordre structurel.

12. Contributeurs de Wikipédia (Dernière mise à jour: $1^{\text {er }}$ juin 2009): Vocabulaire de l'islam. Wikipédia, l'encyclopédie libre. Consulté le 17 juillet 2017, <https://fr.wikipedia.org/w/index. php?title=Vocabulaire_de_l\%27islam\&oldid=41552158>.

13. Buenodic. Consulté le 17 juillet 2017, <http://it.buenodic.com/dunia>.

14. C'est nous qui soulignons les unités confrontées dans notre analyse.

15. Variante: «Rendent plus torturant, / De la velouter, mon feu clos».

16. Variante: «Capricieuse Croate nuit brillante».

17. Daniel Arasse entend par figure composite une figure «composée avec des traits appartenant à différentes figures. C'est le fruit d'une condensation» (Arasse 2000: 107-108).

18. Ungaretti préfère la variante des deux vers, suggérée par Jaccottet (voir note 15).

19. Fla BBI, Lorenzo (Dernière mise à jour : 22 novembre 2006): Un suono di Ungaretti et Jaccottet. Il traduttore di poesia come imitatore. Sguardamobile. Consulté le 18 janvier 2018, <http://www. sguardomobile.it/spip.php?article246>.

20. "[...] in un fitto reticolo di sibilanti, così che l'idea del tondo sia data non dalla stasi della circonferenza, ma dal movimento sinuoso del cerchio nel suo formarsi. É così che il fonosimbolismo è limitato, salvo, translato.» 


\section{RÉFÉRENCES}

Arasse, Daniel (2000): On n'y voit rien. Descriptions. Paris: Gallimard.

Benjamin, Walter (1923/2000): La tâche du traducteur. (Traduit de l'allemand par Maurice de Gandillac) In: Walter Benjamin. Euvres. Tome I. Paris: Gallimard, 142-165.

Berman, Antoine (1995): Pour une critique des traductions: John Donne. Paris: Gallimard.

Berman, Antoine (1999): La traduction et la lettre ou l'auberge du lointain. Paris: Seuil.

Blanchot, Maurice (1971): L’amitié. Paris: Gallimard.

BollaCK, Jean (2000): Sens contre sens. Comment lit-on? Vénissieux: Éditions La passe du vent.

CATFORD, John Cunnison (1978): A Linguistic Theory of Translation: an essay in Applied Linguistics. Oxford: Oxford University Press.

Derrida, Jacques (1982): Réponse à l'intervention de François Péraldi: Le faux sens. In: Claude LÉvesque et Christie V. McDonald, dir. L'oreille de l'autre. Otobiographies, transferts, traductions. Textes et débats avec Jacques Derrida. Montréal: VLB éditeur, 173-178.

DERrIDA, Jacques (1987): Psyché. Inventions de l'autre. Paris: Galilée.

Derrida, Jacques (1996): Le monolinguisme de l'autre ou la prothèse d'origine. Paris: Galilée.

Derrida, Jacques (2004): Qu'est-ce qu'une traduction «relevante»? In: Marie-Louise MaLlet et Ginette Michaud, dir. Jacques Derrida. Paris: Éditions de l'Herne, 561-576.

Graf, Marion, dir. (1998): L'écrivain et son traducteur. Genève: Éditions Zoé.

Guastella, Andrea (2003): Il futuro della memoria. Tre studi su Ungaretti [L'avenir de la mémoire. Trois études sur Ungaretti]. Catane: CUECM.

Humboldt, Wilhelm (von) (2006): Introduction à l'Agamemnon d'Eschyle. (Traduit de l'allemand par Marc de Launay) In: Marc de Launay. Qu'est-ce que traduire? Paris: Vrin, 81-86.

Jaccottet, Philippe (1973): Notes. In: Giuseppe Ungaretti. Vie d'un homme. (Traduit de l'italien par Philippe Jaccottet, Pierre Jean Jouve, Jean Lescure, André Pieyre de Madiargues, Francis Ponge et Armand Robin) Paris: Minuit/Gallimard, 331-338.

JАссоттет, Philippe (1982a): Entretien avec Pascal Ruffenach. Magazine littéraire. 363:10.

Jассоттет, Philippe (1982b) : Postface. In: Homère. L'Odyssée. Paris: La Découverte, 401-410.

JАссоттет, Philippe (1997): Entretien avec Marion Graf. Journal de Genève. 3:30.

Jассоттет, Philippe (2015): Une transaction secrète. Lectures de poésie. Paris: Gallimard.

Kristeva, Irena (2009): Pour comprendre la traduction. Paris: L'Harmattan.

Ladmiral, Jean-René (1998): Principes philosophiques de la traduction. In: Jean-François Mattéi, dir. Le Discours philosophique. Paris: PUF, 977-998.

Ladmiral, Jean-René (2014): Sourcier ou cibliste. Paris: Les Belles Lettres.

Meschonnic, Henri (1999): Poétique du traduire. Lagrasse: Verdier.

Meschonnic, Henri (2007): Éthique et politique du traduire. Lagrasse: Verdier.

Mounin, Georges (1955/1994): Les belles infidèles. Lille: Presses universitaires de Lille.

Отто, Rudolf (2001): Le Sacré. L'élément non rationnel dans l'idée du divin et sa relation avec le rationnel. (Traduit de l'allemand par André JundT) Paris: Payot.

Pergnier, Maurice (1973): Traduction et théorie linguistique. Études de linguistique appliquée. 12:26-38.

Pergnier, Maurice (1980): Les fondements sociolinguistiques de la traduction. Paris: Honoré Champion.

Petrilli, Susan (2001): Il carattere intersemiotico del tradurre [Le caractère intersémiotique du traduire]. Athanor. 4:9-18.

RAO, Sathya (2007): Sujet et traduction. De la décision de Ladmiral à la pulsion de Berman. Meta. 52(3):477-483.

Ricceur, Paul (1977): Herméneutique de l'idée de Révélation. In: Paul Ricceur, Emmanuel Levinas, Edgar Haulotte et al. La révélation. Bruxelles: Facultés universitaires SaintLouis, 15-54.

Schleiermacher, Friedrich (1813/1999): Des différentes méthodes du traduire. (Traduit de l'allemand par Antoine Berman) Paris: Seuil.

Simeone, Bernard (2014) : Écrire, traduire, en métamorphose. Lagrasse: Verdier. 
Starobinski, Jean (1988): Philippe Jaccottet à la recherche de l'insaisissable. Forum der Schriftseller. 2:37-43.

Suchet, Myriam (2009): Outils pour une traduction postcoloniale. Paris: Éditions des archives contemporaines.

Tophoven, Elmar (1995): La traduction transparente. TransLittérature. 10:19-27.

Woolf, Virginia (2008): Orlando: A Biography. Oxford/New York: Oxford University Press.

\section{ANNEXES}

Annexe 1: CEuvres de Giuseppe Ungaretti

Ungaretti, Giuseppe (1916): Il Porto sepolto [Le port enseveli]. Udine: Stabilimento Tipografico Friulano.

Ungaretti, Giuseppe (1916/1919): Allegria di naufragi [Allégresse des naufrages]. 2e éd. Florence: Vallecchi.

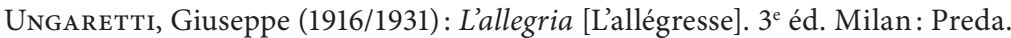

Ungaretti, Giuseppe (1916/1936): L’allegria [L'allégresse]. 4éd. Rome: Novissima.

Ungaretti, Giuseppe (1916/1942): Vita d'un uomo [Vie d'un homme]. Vol. I. L'allegria [L'allégresse]. $5^{\mathrm{e}}$ éd. Milan: Arnoldo Mondadori Editore.

Ungaretti, Giuseppe (1933): Sentimento del Tempo [Sentiment du temps]. Florence/Rome: Vallecchi/ Novissima.

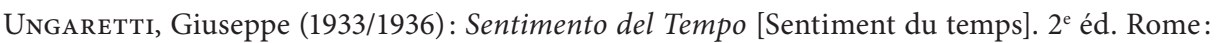
Novissima.

Ungaretti, Giuseppe (1933/1943): Vita d'un uomo [Vie d'un homme]. Vol. II. Sentimento del

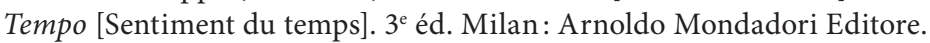

Ungaretti, Giuseppe (1947): Vita d'un uomo [Vie d'un homme]. Vol. IV. Il Dolore [La douleur]. Milan: Arnoldo Mondadori Editore.

Ungaretti, Giuseppe (1950): La Terra Promessa [La terre promise]. Milan: Arnoldo Mondadori Editore.

Ungaretti, Giuseppe (1950/1953): Vita d'un uomo [Vie d'un homme]. Vol. VIII. La Terra Promessa [La terre promise]. $2^{\mathrm{e}}$ éd. Milan: Arnoldo Mondadori Editore.

Ungaretti, Giuseppe (1960): Il Taccuino del Vecchio [Le carnet du vieillard]. Milan: Arnoldo Mondadori Editore.

Ungaretti, Giuseppe (1960/1961): Vita d'un uomo [Vie d'un homme]. Vol. VII. Il Taccuino del Vecchio [Le carnet du vieillard]. $2^{2}$ éd. Milan: Arnoldo Mondadori Editore.

Ungaretti, Giuseppe (1969a): Vita d'un uomo: tutte le poesie [Vie d'un homme: tous les poèmes]. (Dirigé par Leone Piccioni) I Meridiani. Milan: Arnoldo Mondadori Editore.

Ungaretti, Giuseppe (1969b): Le bocche di Cattaro [Le livre de Cattaro]. In: Leone Piccioni, dir. Vita d'un uomo: tutte le poesie [Vie d'un homme: tous les poèmes]. I Meridiani. Milan: Arnoldo Mondadori Editore, 324.

Ungaretti, Giuseppe (1969c): Dunja [Dunja]. In: Leone Piccioni, dir. Vita d'un uomo: tutte le poesie [Vie d'un homme: tous les poèmes]. I Meridiani. Milan: Arnoldo Mondadori Editore, 325.

Ungaretti, Giuseppe (1970a): L'impietrito e il velluto [Le pétrifié et le velours]. Roma: Grafica Romero.

Ungaretti, Giuseppe (1970b): L'impietrito e il velluto [Le pétrifié et le velours]. In: Leone Piccioni, dir. (1969/1970) Vita d'un uomo: tutte le poesie [Vie d'un homme: tous les poèmes]. $2^{\mathrm{e}}$ éd. I Meridiani. Milan: Arnoldo Mondadori Editore, 326.

\section{Annexe 2: CEuvres et traductions de Philippe Jaccottet}

Góngora y Argote, Luis (de) (1613/1984): Les Solitudes. (Traduit de l'espagnol par Philippe JАСсоттет) Chêne-Bourg: La Dogana. 
Góngora y Argote, Luis (de) (xvi-XviI s./1985): Treize sonnets et un fragment. (Traduit de l'espagnol par Philippe Jассоттет) Chêne-Bourg: La Dogana.

Hölderlin, Friedrich (1797/1957): Hypérion ou l'Ermite de Grèce. (Traduit de l'allemand par Philippe Jассоттет) Lausanne: Mermod.

Homère (viII s. av. J.-C./1982): L’Odyssée. (Traduit du grec ancien par Philippe JAccottet) Paris: La Découverte.

Jассоттет, Philippe (1957): La Promenade sous les arbres. Lausanne: Mermod.

Jассоттет, Philippe (2009): L'encre serait de l'ombre. Paris: Gallimard.

Mandelstam, Ossip (1908-1937/1994): Simple promesse (choix de poèmes 1908-1937). (Traduit du russe par Philippe Jaccottet, Louis Martinez et Jean-Claude Schneider) ChêneBourg: La Dogana.

Mann, Thomas (1912/1947): La Mort à Venise. (Traduit de l'allemand par Philippe Jacсоттет) Lausanne: Mermod.

Mann, Thomas (1949): Études: Goethe - Nietzsche - Joseph et ses frères. (Traduit de l'allemand par Philippe Jассоттет) La Grenade. Lausanne: Mermod.

MusiL, Robert (1930/1957): L'Homme sans qualités. (Traduit de l'allemand par Philippe JAccotTET) Paris: Seuil.

MusiL, Robert (1906/1960): Les Désarrois de l'élève Törless. (Traduit de l'allemand par Philippe JACсоттет) Paris: Seuil.

Musil, Robert (1911/1962): Noces. In: Robert MusiL. Trois femmes, suivi de Noces. (Traduit de l'allemand par Philippe Jассоттет) Paris: Seuil.

Musil, Robert (1924/1962): Trois femmes. In: Robert Musil. Trois femmes, suivi de Noces. (Traduit de l'allemand par Philippe Jacсоттет) Paris: Seuil.

Musil, Robert (1935/1965): Euvres pré-posthumes. (Traduit de l'allemand par Philippe JACcoTTET) Paris: Seuil.

Musil, Robert (1976/1981) : Journaux. (Traduit de l'allemand par Philippe JАссоттет) Don des langues. Paris: Seuil.

Musil, Robert (1978/1984): Essais, conférences, critique, aphorismes, réflexions. (Textes choisis et traduits de l'allemand par Philippe Jассоттет) Don des Langues. Paris: Seuil.

MusiL, Robert (1921/1985): Les Exaltés. In: Robert Musil. Théâtre: Les Exaltés - Vincent et l'amie des personnalités - Prélude au mélodrame «Le zodiaque». (Traduit de l'allemand par Philippe Jaccottet) Paris: Seuil.

MusiL, Robert (1924/1985): Vincent et l'amie des personnalités. In: Robert MusiL. Théâtre: Les Exaltés - Vincent et l'amie des personnalités - Prélude au mélodrame "Le zodiaque». (Traduit de l'allemand par Philippe Jассоттет) Paris: Seuil.

MusiL, Robert (1918/1985): Prélude au mélodrame «Le zodiaque». In: Robert MusiL. Théâtre: Les Exaltés - Vincent et l'amie des personnalités - Prélude au mélodrame "Le zodiaque». (Traduit de l'allemand par Philippe Jассоттет) Paris: Seuil.

MusiL, Robert (1981/1987): Lettres choisies. (Traduit de l'allemand par Philippe Jaccottet) Don des langues. Paris: Seuil.

Musil, Robert (1978/1989): Proses éparses. (Textes choisis et traduits de l'allemand par Philippe Jассоттет) Don des langues. Paris: Seuil.

MusiL, Robert (1930/2011): L’Homme sans qualités. (Traduit de l'allemand par Philippe JACCOTTET) Tome I. $2^{e}$ éd. Paris: Seuil.

MusiL, Robert (1930/2011): L'Homme sans qualités. (Traduit de l'allemand par Philippe JACcoTtet, Jean-Pierre Cometti et Marianne Rocher-Jacquin) Tome II. $2^{\mathrm{e}}$ éd. Paris: Seuil.

RiLke, Rainer Maria (1976): CEuvres. Vol. III. Correspondance. (Traduit de l'allemand par Blaise Briod, Philippe Jaccottet et Pierre Klossowski) Paris: Seuil.

Rilke, Rainer Maria et Andreas-Salomé, Lou (1988): Correspondance. Lou Andreas-Salomé, Rainer Maria Rilke. (Traduit de l'allemand par Philippe Jассоттет) Paris: Seuil.

Ungaretti, Giuseppe (1969/1973a): Croatie secrète. In: Giuseppe Ungaretti. Vie d'un homme: poésie, 1914-1970. (Traduit de l'italien par Philippe Jacсотtet, Pierre Jean Jouve, Jean 
164 MetA, LXIV, 1, 2019

Lescure, André Pieyre de Madiargues, Francis Ponge et Armand Robin) Paris: Gallimard/Éditions de Minuit, 340.

Ungaretti, Giuseppe (1969/1973b): Dunja. In: Giuseppe Ungaretti. Vie d'un homme: poésie, 1914-1970. (Traduit de l'italien par Philippe Jaccottet, Pierre Jean Jouve, Jean Lescure, André Pieyre de Madiargues, Francis Ponge et Armand Robin) Paris: Gallimard/Éditions de Minuit, 341-342.

Ungaretti, Giuseppe (1970/2005): Le pétrifié et le velours. In: Giuseppe Ungaretti. Vie d'un homme: poésie, 1914-1970. (Traduit de l'italien par Philippe Jaccottet, Pierre Jean Jouve, Jean Lescure, André Pieyre de Madiargues, Francis Ponge et Armand Robin) Paris: Gallimard/Éditions de Minuit, 328-329. 